\title{
Free radicals: definition, causes, their role in diabetes and treatment
}

\author{
Shimaa Mohammed EL Shafei ,Iman Ahmed Mohammed \\ Shimaa.2020528@stemmenof.moe.edu.eg \\ eman.2020506@stemmenof.moe.edu.eg \\ Student at Menofia STEM school, EL bajour, Menofia ,32821,Egypt \\ Student at Menofia STEM school, First of October, Giza, 12573, Egypt
}

\begin{abstract}
This research aims to mention how free radicals play a vital role in our body and gradually cause diabetes mellitus. According to studies, free radicals and oxidative stress play an essential role in the development of type 1and type 2 Diabetes. The human body is affected with diabetic Mellitus through beta-cell damage that may be the result of oxidative stress. As free radicals are highly reactive molecules including reactive oxygen species (ROS) and reactive nitrogen species (RNS). They disrupt the function of lipid, protein, DNA and the imbalance between them and antioxidants causes oxidative stress. Increased levels of oxidative stress in our body tissue and blood is considered to play a critical role in diabetes mellitus.

Key words: free radicals; oxidative stress; antioxidant; reactive oxygen species (ROS); reactive nitrogen species; (RNS) diabetes mellitus
\end{abstract}

\section{Introduction}

Free radicals are molecules or molecular fragments containing a single unpaired electron. Free radicals are derived either from normal essential metabolic processes in the human body or from external sources. They play a dual role in the human body as both toxic and beneficial compounds since they can be either harmful or helpful to the body. There are many types of free radicals in humans. The most significant are oxygen free radicals (reactive oxygen species) and nitrogen free radicals (reactive nitrogen species). There must be a balance between free radical radicals and antioxidants to protect our body cells against FR and prevent oxidative stress. Antioxidants are known as free radical scavengers. They are chemicals that interact with and neutralize free radicals. One of the main consequences of oxidative stress is genomic instability (DNA damage), which includes base modifications and strand breaks.Oxidatively induced DNA damage is repaired in living cells by different pathways. Unrepaired and accumulated DNA lesions may lead to disease processes such as diabetes.

\section{$\underline{\text { (1.0) Free radicals definition }}$}

Free radicals are known as atoms or molecules containing one or more unpaired electrons in a valence shell. They are highly reactive because they have free electrons. The odd number of electron(s) of a free radical makes it unstable, short-lived, and highly reactive. Because of their high reactivity, they can abstract electrons from other compounds to attain stability. Thus the attacked molecule loses its electron and becomes a free radical itself, beginning a chain reaction cascade that finally damages the living cell. It's believed that approximately 10000-20000 free radicals are invading most of the cells every day. Endogenous sources such as (Mitochondria transport chain leakage) and exogenous sources such as (Drugs, pollution, and radiation) cause free radical-induced DNA damage in living organisms by different mechanisms.

Many of these radicals are beneficial because they work for immune cells responsible for killing bacterial cells and toning smooth muscles. They regulate the normal working of blood vessels and internal organs. 
Although FRs can help fight off pathogens that lead to infections, they also react with biomolecules, including DNA and protein. It causes DNA damage as shown in figure (1.0), and this damage is called oxidative damage. Free radicals are associated with human diseases, including diabetes and many others.

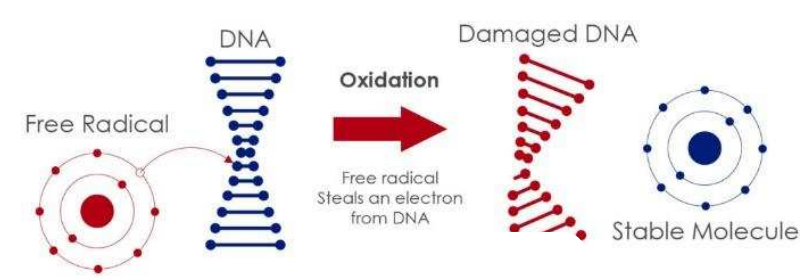

Figure (1.0)

\section{(2.0) Most common types of free radicals}

\section{Reactive oxygen species (Ros)}

There are many types of radicals, but those of most concern in biological systems are derived from oxygen and known collectively as reactive oxygen species.

Oxygen has a unique molecular structure and is abundant within cells. Atomic oxygen has two unpaired electrons in separate orbits in its outer electron shell so that it readily accepts free electrons generated by normal oxidative metabolism within the cell. The electron structure makes oxygen susceptible to radical formation. The sequential reduction of oxygen through the addition of electrons leads to the formation of many ROS such as superoxide, hydrogen peroxide, hydroxyl radical.

Most reactive oxygen species can generate as by-products during the mitochondrial electron transport chain (ETC) or from the normal oxidation-reduction process in the body. Oxidative reactions in our bodies usually ensure that molecular oxygen is reduced completely to water. In Normal, four electrons are transferred to molecular oxygen so that it is completely reduced to a water molecule. But the incomplete reduction of oxygen leads to the production of specific reactive oxygen species by single electron additions (e-) such as superoxide anion, hydrogen peroxide, and hydroxyl radical as shown in figure (2.0).

(a)Superoxide radicals are produced when a single electron is transferred to oxygen, and it is the least powerful Ros.

(b)Hydrogen peroxide radical is the two-electron reduction product of oxygen.

(c)Hydroxyl radical is the three electron reduction product of oxygen, and it is the most powerful oxygenfree radical (Ros).

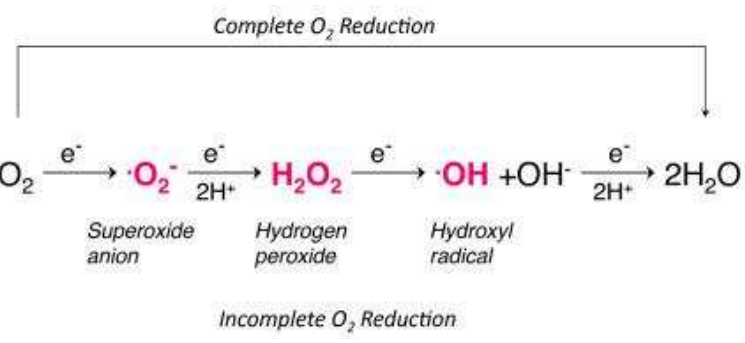

Figure (2.0)

ROS can be formed by cellular respiration, by lipoxygenases (LOX) and cyclooxygenases (COX) during the arachidonic acid metabolism, and by endothelial and inflammatory cells.

They are produced by other pathways as well, including the respiratory burst taking place in activated phagocytes, ionizing radiation's damaging effect on components of cell membranes.

ROS are beneficial to cells, supporting basic cellular processes and viability. Maintaining a basal level of ROS in cells is essential for life. However, they also damage lipids, DNA, RNA, and proteins, which, in theory, contribute to the physiology of aging and many diseases like diabetes. 


\section{Reactive nitrogen species (RNS)}

(RNS) are various nitric oxide-derived compounds, including nitroxyl anion, nitrosonium cation, higher oxides of nitrogen, S-nitrosothiols, and dinitrosyl iron complexes.

Reactive nitrogen species (RNS) are a subset of free oxygen radicals called reactive oxygen species (ROS). Reactive oxygen species (ROS) and reactive nitrogen species (RNS) targeting mitochondria are major causative factors in disease pathogenesis .

RNS have been recognized as playing an important role in the physiologic regulation of many such as smooth muscle cells, cardiomyocytes, platelets, and nervous and juxtaglomerular cells .

However, they can causes damage to the cell and causes many diseases. Usually, these species are removed rapidly before they cause cell dysfunction and death.

\section{(3.0) Diabetes mechanism}

Diabetes mellitus (DM), commonly known as diabetes, is a metabolic disease that causes high blood sugar levels over a prolonged period. With type 1 diabetes, the body does not make insulin. With type 2 diabetes, the more common type, the body does not make or use insulin well. The hormone insulin moves sugar from the blood into body cells to be stored or used for energy. Without enough insulin, the glucose stays in the blood and causes diabetes. Gestational diabetes mellitus (GDM) is the third main form and occurs when pregnant women without a previous history of diabetes develop a high blood glucose level.

\section{(3.1) there are many reasons which lead to diabetes and its types:}

Type I diabetes also known as autoimmune diabetes is a chronic disease characterized by insulin deficiency due to pancreatic $\beta$-cell loss and leads to hyperglycemia.it occurs when the body's immune system destroys the cells in the pancreas that produce insulin. And after researching, we also think type 1 diabetes is caused by free radicals, genes, and environmental factors. Type I diabetes is usually diagnosed in children and young people, so it used to be called juvenile diabetes.

Type II diabetes occurs when the body either does not produce enough insulin or specific cells are not responding to insulin (insulin resistance). Also, Type 2 diabetes, the most common form of diabetes, is caused by several factors, including lifestyle factors and genes. Free radical also has a pivotal role in causing diabetes type 2 . Type 2 diabetes also used to be known as adult-onset diabetes, but the increase in the number of children with obesity has led to more cases of type 2 diabetes in younger people.

And finally, gestational diabetes mellitus (GDM) starts when the mother's body is not able to make and use all the insulin it needs for pregnancy. Women who are overweight or obese may already have insulin resistance when they become pregnant. it resembles type 2 diabetes in several respects, involving a combination of relatively inadequate insulin secretion and responsiveness.

The high blood sugar produces the symptoms of frequent urination, increased thirst, and increased hunger, weight loss, and fatigue .

Untreated diabetes can cause many complications. Acute complications include diabetic ketoacidosis and nonketotic hyperosmolar coma. Serious long-term complications include heart disease, stroke, kidney failure, foot ulcers, and damage to the eyes (blindness).

Glucose tests can detect diabetes in very early stages before symptoms appear.

\section{Diabetes and oxidative stress}

Diabetes eventually affects the function of most, if not all, tissues in the body. Thus, it is reasonable to consider a basic mechanism, such as oxidative stress, as a possible common cause of the diverse complications of diabetes.

However, oxidative stress has a primary role in the pathogenesis of diabetes whether it is a secondary indicator of end-stage tissue damage in diabetes. The increase in glycoxidation and lipoxidation products in plasma and tissue proteins suggests that oxidative stress is increased in diabetes defenses. 
Increasing evidence in both experimental and clinical studies suggests that there is a close link between hyperglycemia, oxidative stress, and diabetic complications. Oxidative stress is a phenomenon caused by an imbalance between the production and accumulation of oxygen reactive species (ROS) in cells and tissues and the ability of a biological system to detoxify these reactive products.

As shown in figure (3.0) Oxidative stress and oxidative damage to tissues are common endpoints of chronic diseases including diabetes.

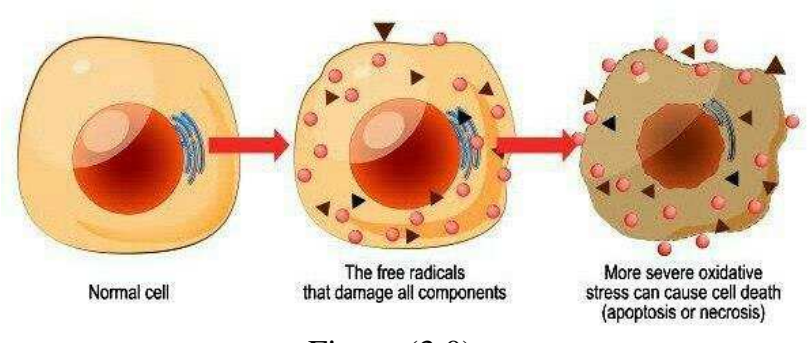

Figure (3.0)

So, there must be a balance between free radical radicals and antioxidants to protect our body cells against FR. But when there is an imbalance between these two, oxidative stress occurs. From all of that, we see that the antioxidant can be the reason to cure oxidative stress and many diseases diabetes.

\section{Antioxidant and free radical scavengers}

Antioxidants are substances that can prevent or slow damage to cells caused by free radicals. They act as radical scavengers and helps in converting the radicals to less reactive species.

Antioxidants neutralize free radicals by giving up some of their electrons as shown in figure (4.0). In making this sacrifice, they act as a natural "off" switch for the free radicals. That helps break a chain reaction that can affect other molecules in the cell and other cells in the body.

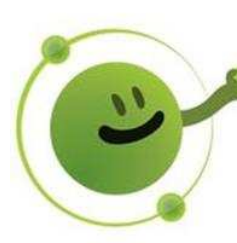

Antioxidant

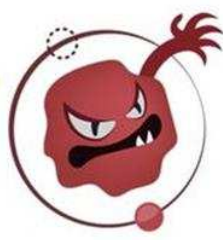

Free radical Figure (4.0)

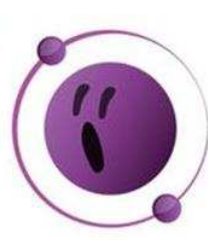

Healthy cell

As free radical scavengers (FRS) either prevent reactive oxygen species from being formed or remove them before they can damage vital components of the cell so they are known as preventive and chainbreaking antioxidants.

\section{(5.1) Endogenous anti-oxidants (non enzymatic)}

Several studies have addressed the possible participation of dietary antioxidants, such as vitamins $\mathrm{C}$ and E, selenium, Beta carotene, other carotenoids, and oxycarotenoids, e.g., lycopene and luteinare in ameliorating the diabetic state and retarding the development of diabetes complications. A variety of antioxidants is found in fruit, vegetables, and many other resources.

\section{(5.2) Endogenous antioxidants (enzymatic)}

Endogenous antioxidants are enzymes made by our body. They can be categorized into primary antioxidants and secondary antioxidants

The first group is primary antioxidants, they so-called chain-breaking antioxidants. They can react directly with free radicals by transforming them into more stable, non-radical products. Hence, primary antioxidants play an important role in lipid oxidation because they can react with the formed lipid radicals and convert them into non-radicals and thereby hinder further decomposition of the lipids.

They include superoxide dismutase (SOD), peroxidase, and catalase i.e. enzymatic mechanism of inactivation of ODFR (oxygen-derived free Radicals).

As shown in figure (5.0), Superoxide dismutase (SOD) catalyzed dismutation (partitioning) of 2 molecules of superoxide anion O2. Producing hydrogen peroxide.

Peroxides produced by (SOD) are also toxic. So they are detoxified by conversion to water via the enzyme peroxidase.

Catalase is a heme-containing enzyme that catalyses the dismutation of hydrogen peroxide into water and oxygen.

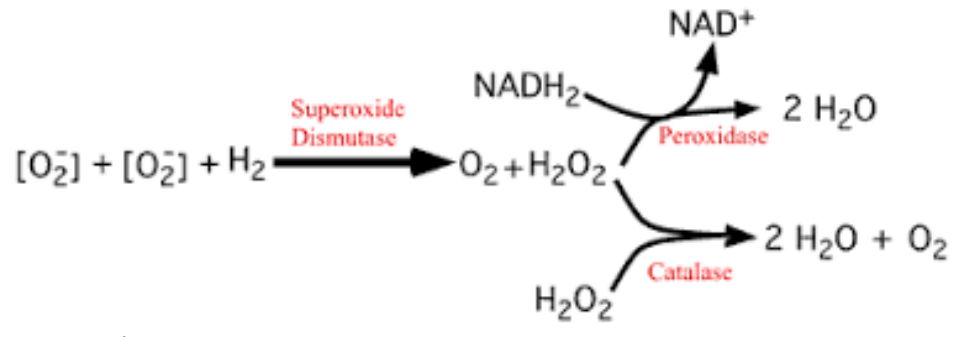

Figure (5.0) 
The second group is secondary, or preventive, antioxidants that work indirectly on limiting lipid oxidation. Several mechanisms including the chelation of transition metals, singlet-oxygen quenching (in photo-oxidation), and oxygen scavenging can be exhibited by these secondary antioxidants. Furthermore, some secondary antioxidants can work synergistically by regenerating primary antioxidants and thereby restore the antioxidant activity of primary antioxidants to ensure their continuous antioxidant activity.

They include ascorbic acid Glutathione reductase, glucose-6-phosphate dehydrogenase, glutathione-stransferase, and ubiquinone.

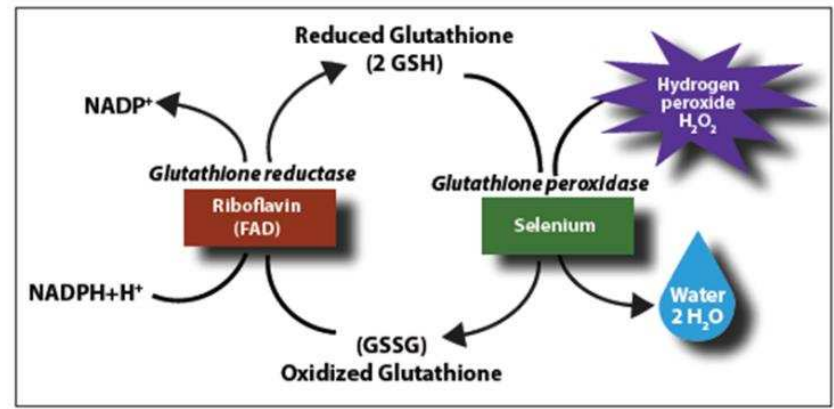

Figure (6.0)

Figure (6.0): The Glutathione Redox Cycle. Reduced Glutathione (GSH) reduces oxidants with the aid of selenium-containing glutathione peroxidase (GPx), forming oxidized glutathione (GSSG) in the process. Glutathione reductase converts GSSH back to GSH to complete the cycle, which is repeated as needed. An alternative pathway for reducing GSSH utilizes thioredoxin reductase along with thioredoxin (Trx) and NADPH to reduce the disulfide bonds.

\section{Conclusion}

As we mentioned that FR species which can lead to cause oxidative stress react with biomolecules in cells, including DNA and causes damage to it. The resulting damage to DNA, which is also called oxidative damage, leads to many diseases because DNA damage causes chemical modification of amino acids in proteins. Then, these proteins are recognized as non-self by the immune system so the immune system will produce anti-bodies to damage these proteins in different cells and this is called autoimmune disease.

In the same way, that is what happens in the beta-cell which makes insulin. The immune system attacks this cell due to the lack of identifying it by the body, and there is no doubt that oxidative stress has a central role in this happening. From all of that, there must be a balance between FRS and antioxidants to protect the body and its DNA from damage caused by free radicals and oxidative stress.

\section{Reference}

1. R;, M. M. C. A. (n.d.). Reactive nitrogen species: Molecular mechanisms and potential significance in health and disease. Antioxidants \& redox signaling. https://pubmed.ncbi.nlm.nih.gov/19014277/

2. https://vitashell.co.za/blogs/blog/understanding-free-radicals Understanding free radicals. VitaShell. (n.d.).

3. Passwater, R. A., By, -, Passwater, R. A., \& Dr. Richard Passwater is the author of more than 45 books and 500 articles on nutrition. Dr. Passwater has been WholeFoods Magazine's science editor and author of this column since 1984. More information is available on his web site. (2016, June 14). Longer lifespans and better health with glutathione: Taking the confusion out of the master antioxidant. WholeFoods Magazine. https://wholefoodsmagazine.com/columns/vitaminconnection/longer-lifespans-and-better-health-glutathione-taking-confusion-out-maste

4. Mehta, S. K., \& Gowder, S. J. T. (2015, November 11). Members of antioxidant machinery and their functions. IntechOpen. https://www.intechopen.com/chapters/49498 
5. Antioxidant. Antioxidant - an overview | ScienceDirect Topics. (n.d.). https://www.sciencedirect.com/topics/earth-and-planetary-sciences/antioxidant

6. EN;, F. (n.d.). The antioxidant and nutritional effects of tocopherols, ascorbic acid and betacarotene in relation to processing of edible oils. Bibliotheca nutritio et dieta. https://pubmed.ncbi.nlm.nih.gov/2658965

7. Giacco, F., \& Brownlee, M. (2010, October 29). Oxidative stress and diabetic complications. Circulation research. https://www.ncbi.nlm.nih.gov/pmc/articles/PMC2996922

8. Johansen, J. S., Harris, A. K., Rychly, D. J., \& Ergul, A. (2005, April 29). Oxidative stress and the use of antioxidants in diabetes: Linking basic science to clinical practice. Cardiovascular Diabetology. https://cardiab.biomedcentral.com/articles/10.1186/1475-2840-4-5

9. Asmat, U., Abad, K., \& Ismail, K. (2016, September). Diabetes mellitus and oxidative stress-a concise review. Saudi pharmaceutical journal : SPJ : the official publication of the Saudi Pharmaceutical Society. https://www.ncbi.nlm.nih.gov/pmc/articles/PMC5059829/

10. MediLexicon International. (n.d.). What is oxidative stress? Effects on the body and how to reduce. Medical News Today. https://www.medicalnewstoday.com/articles/324863\#conditions

11. Pizzino, G., Irrera, N., Cucinotta, M., Pallio, G., Mannino, F., Arcoraci, V., Squadrito, F., Altavilla, D., \& Bitto, A. (2017). Oxidative stress: Harms and benefits for human health. Oxidative medicine and cellular longevity. https://www.ncbi.nlm.nih.gov/pmc/articles/PMC5551541/

12. Moore, M. (2020, September 30). Oxidative stress and inflammation: Causes, effects, and prevention. Life Science product | Helvetica Health Care. https://www.h-h-c.com/oxidative-stressand-inflammation/

13. WebMD. (n.d.). Type 2 Diabetes: Symptoms, Causes, diagnosis, and treatment. WebMD. https://www.webmd.com/diabetes/type-2-diabetes

14. NHS. (n.d.). Nhs choices. https://www.nhs.uk/conditions/type-2-diabetes

15. Mayo Foundation for Medical Education and Research. (2020, October 30). Diabetes. Mayo Clinic. https://www.mayoclinic.org/diseases-conditions/diabetes/symptoms-causes/syc-20371444

16. Melissa Conrad Stöppler, M. D. (2019, October 31). 9 symptoms of type $1 \&$ type 2 Diabetes: Complications, causes \& diet. MedicineNet. https://www.medicinenet.com/diabetes_mellitus/article.htm

17. Watson, S. (2020, February 27). Diabetes: Symptoms, causes, treatment, prevention, and more. Healthline. https://www.healthline.com/health/diabetes\#symptoms

18. Rns definition. IG. (n.d.). https://www.ig.com/en/glossary-trading-terms/rns-definition

19. R;, M. M. C. A. (n.d.). Reactive nitrogen species: Molecular mechanisms and potential significance in health and disease. Antioxidants \& redox signaling. https://pubmed.ncbi.nlm.nih.gov/19014277/

20. Reactive oxygen and nitrogen species - ppt video online download. SlidePlayer. (n.d.). https://slideplayer.com/slide/5337694/

21. Superoxide dismutase (sod) \& glisodin. FULL HEALTH SECRETS. (n.d.). https://www.fullhealthsecrets.com/remedies/supplements/superoxide-dismutase-sod-glisodin/

22. Scavenger. Scavenger - an overview $\mid$ ScienceDirect Topics. (n.d.). https://www.sciencedirect.com/topics/pharmacology-toxicology-and-pharmaceuticalscience/scavenger.

23. Carmeli, E., Coleman, R., \& Berner, Y. N. (2004). Activities of antioxidant scavenger enzymes (superoxide dismutase and glutathione peroxidase) in erythrocytes in adult women with and without type ii diabetes. Experimental diabesity research. https://www.ncbi.nlm.nih.gov/pmc/articles/PMC2496882

24. Libretexts. (2020, August 11). 5.5. the FREE-RADICAL chain reaction. Chemistry LibreTexts. https://chem.libretexts.org/Courses/Purdue/Purdue_Chem_26100\%3A_Organic_Chemistry_I_(We nthold)/Chapter_05\%3A_The_Study_of_Chemical_Reactions/5.5.\%09The_Free-

Radical_Chain_Reaction.

25. Oxygen specie. Oxygen Specie - an overview | ScienceDirect Topics. (n.d.). https://www.sciencedirect.com/topics/engineering/oxygen-specie

26. National Institutes of Health. (n.d.). U.S. national library of medicine. https://openi.nlm.nih.gov/detailedresult?img=PMC3219237_cc9185-2\&req=4.

27. Ma, R., Lin, G., Zhou, Y., Liu, Q., Zhang, T., Shan, G., Yang, M., \& Wang, J. (2019, July 19). A review of oxygen reduction mechanisms for metal-free carbon-based electrocatalysts. Nature News. https://www.nature.com/articles/s41524-019-0210-3. 
28. Krumova, K., \& Cosa, G. (n.d.). Chapter 1 overview of reactive oxygen species. Royal Society of Chemistry. https://pubs.rsc.org/en/content/chapterhtml/2016/bk9781782620389-00001?isbn=9781-78262-038-9

29. Reactive oxygen species. Reactive Oxygen Species - an overview | ScienceDirect Topics. (n.d.). https://www.sciencedirect.com/topics/earth-and-planetary-sciences/reactive-oxygen-species

30. Turrens, J. F. (2003, October 15). Mitochondrial formation of reactive oxygen species. The Journal of physiology. https://www.ncbi.nlm.nih.gov/pmc/articles/PMC2343396/

31. Phaniendra, A., Jestadi, D. B., \& Periyasamy, L. (2015, January). Free radicals: Properties, sources, targets, and their implication in various diseases. Indian journal of clinical biochemistry : IJCB. https://www.ncbi.nlm.nih.gov/pmc/articles/PMC4310837/

32. Li, R., Jia, Z., \& Trush, M. A. (2016). Defining ros in biology and medicine. Reactive oxygen species (Apex, N.C.). https://www.ncbi.nlm.nih.gov/pmc/articles/PMC5921829/

33. Aprioku, J. S. (2013, October). Pharmacology of free radicals and the impact of reactive oxygen species on the testis. Journal of reproduction \& infertility. https://www.ncbi.nlm.nih.gov/pmc/articles/PMC3911811/

34. Das, K., \& Roychoudhury, A. (1AD, January 1). Reactive oxygen species (ros) and response of antioxidants as ros-scavengers during environmental stress in plants. Frontiers. https://www.frontiersin.org/articles/10.3389/fenvs.2014.00053/full.

35. Ray, P. D., Huang, B.-W., \& Tsuji, Y. (2012, May). Reactive oxygen species (ros) homeostasis and redox regulation in cellular signaling. Cellular signalling. https://www.ncbi.nlm.nih.gov/pmc/articles/PMC3454471/

36. Phaniendra, A., Jestadi, D. B., \& Periyasamy, L. (2015, January). Free radicals: Properties, sources, targets, and their implication in various disea ses. Indian journal of clinical biochemistry : IJCB. https://www.ncbi.nlm.nih.gov/pmc/articles/PMC4310837/

37. Lynne Eldridge, M. D. (2020, February 2). What exactly are free radicals and Why are they important? Verywell Health. https://www.verywellhealth.com/information-about-free-radicals2249103

38. Di Meo, S., Reed, T. T., Venditti, P., \& Victor, V. M. (2016, July 12). Role of ros and rns sources in physiological and pathological conditions. Oxidative Medicine and Cellular Longevity. https://www.hindawi.com/journals/omcl/2016/1245049/

39. Admin. (2020, December 8). What are free radicals? - definition, examples, types, mechanism \& uses. BYJUS. https://byjus.com/chemistry/free-radicals/

40. Reactive nitrogen Species. Reactive Nitrogen Species - an overview | ScienceDirect Topics. (n.d.). https://www.sciencedirect.com/topics/medicine-and-dentistry/reactive-nitrogen-species

41. A;, F. B. H. (n.d.). Reactive oxygen species (ros) and reactive nitrogen species (rns) generation by silica in inflammation and fibrosis. Free radical biology \& medicine. https://pubmed.ncbi.nlm.nih.gov/12788471/

42. Phaniendra, A., Jestadi, D. B., \& Periyasamy, L. (2015, January). Free radicals: Properties, sources, targets, and their implication in various diseases. Indian journal of clinical biochemistry : IJCB. https://www.ncbi.nlm.nih.gov/pmc/articles/PMC4310837/

43. Lobo, V., Patil, A., Phatak, A., \& Chandra, N. (2010, July). Free radicals, antioxidants and functional foods: Impact on human health. Pharmacognosy reviews. https://www.ncbi.nlm.nih.gov/pmc/articles/PMC3249911/

44. Asmat, U., Abad, K., \& Ismail, K. (2015, March 21). Diabetes mellitus and oxidative stress-a concise review. Saudi Pharmaceutical Journal. https://www.sciencedirect.com/science/article/pii/S1319016415000766\#: :text=Increase\%20in\%2 0the\%20levels\%20of,diabetes\%20mellitus\%20and\%20its\%20complications

45. Cigliola, V., Ghila, L., Thorel, F., van Gurp, L., Baronnier, D., Oropeza, D., Gupta, S., Miyatsuka, T., Kaneto, H., Magnuson, M. A., Osipovich, A. B., Sander, M., Wright, C. E. V., Thomas, M. K., Furuyama, K., Chera, S., \& Herrera, P. L. (2018, October 22). Pancreatic islet-autonomous insulin and smoothened-mediated signalling modulate identity changes of glucagon $+\alpha$-cells. Nature News. https://www.nature.com/articles/s41556-018-0216-y

46. Hanselmann, F. (n.d.). The natural diabetes cure curing blood sugar disorders without drugs. Academia.edu. https://www.academia.edu/9764015/The_Natural_Diabetes_Cure_Curing_Blood_Sugar_Disorders _Without_Drugs

47. Shouip, H. A. (2014, December 31). (PDF) diabetes mellitus. ResearchGate. https://www.researchgate.net/publication/270283336_Diabetes_mellitus

48. Shouip, H. A. (2014, December 31). (PDF) diabetes mellitus. ResearchGate. https://www.researchgate.net/publication/270283336_Diabetes_mellitus 
49. (Pdf) mechanisms of insulin resistance in obesity. ResearchGate. (n.d.).

https://www.researchgate.net/publication/235885963_Mechanisms_of_insulin_resistance_in_obesi ty

50. (Pdf) guideline for the management of insulin resistance. ResearchGate. (n.d.).

https://www.researchgate.net/publication/283719874_Guideline_for_the_Management_of_Insulin _Resistance

51. (PDF) clinical review: Oxygen as a signaling molecule. ResearchGate. (n.d.). https://www.researchgate.net/publication/47728958_Clinical_review_Oxygen_as_a_signaling_mo lecule

52. (PDF)type1 diabetes mellitus. ResearchGate. (n.d.). https://www.researchgate.net/publication/315903949_Type_1_diabetes_mellitus

53. Mehta, Shalini Kapoor, and Sivakumar Joghi Thatha Gowder. "Members of Antioxidant Machinery and Their Functions." IntechOpen, IntechOpen, 11 Nov. 2015, www.intechopen.com/chapters/49498.

54. 3- Poljsak, Borut, et al. "Achieving the Balance between Ros and Antioxidants: When to Use the Synthetic Antioxidants." Oxidative Medicine and Cellular Longevity, Hindawi, 29 Apr. 2013, www.hindawi.com/journals/omcl/2013/956792/

55. 4- Wu, Danli, and Patricia Yotnda. "Production and Detection of Reactive Oxygen Species (Ros) in Cancers." Journal of Visualized Experiments : JoVE, MyJove Corporation, 21 Nov. 2011, www.ncbi.nlm.nih.gov/pmc/articles/PMC3308605/

56. 5-Pizzino, Gabriele, et al. "Oxidative Stress: Harms and Benefits for Human Health." Oxidative Medicine and Cellular Longevity, Hindawi, 2017, www.ncbi.nlm.nih.gov/pmc/articles/PMC5551541/

57. 6- Martínez, M. Carmen, and Ramaroson Andriantsitohaina. "Reactive Nitrogen Species: Molecular Mechanisms and Potential Significance in Health and Disease." Antioxidants \& Redox Signaling, vol. 11, no. 3, 2009, pp. 669-702., doi:10.1089/ars.2007.1993

58. 7-Kaludercic, Nina, and Valentina Giorgio. "The Dual Function of REACTIVE OXYGEN/NITROGEN Species In Bioenergetics and CELL Death: The Role of ATP Synthase.” Oxidative Medicine and Cellular Longevity, Hindawi, 10 Mar. 2016, www.hindawi.com/journals/omcl/2016/3869610/

59. 8- "Diabetes | Type 1 Diabetes | Type 2 Diabetes." MedlinePlus, U.S. National Library of Medicine, 2 Aug. 2021, medlineplus.gov/diabetes.html.

60. 9-Biochemistry, Department of Chemistry and. "The Role of Oxidative Stress in Diabetic Complications : Current Opinion in Endocrinology, Diabetes and Obesity.” LWW, journals.lww.com/coendocrinology/abstract/1996/08000/the_role_of_oxidative_stress_in_diabetic.1.aspx.

61. 10-Piconi, Ludovica, et al. "Oxidative Stress in Diabetes.” De Gruyter, De Gruyter, 16 Sept. 2003, www.degruyter.com/document/doi/10.1515/CCLM.2003.177/html

62. 11-“Antioxidants: Health Benefits and Nutritional Information.” Medical News Today, MediLexicon International, www.medicalnewstoday.com/articles/301506

63. Hatwalne, Milind S. "Free Radical Scavengers in Anaesthesiology and Critical Care." Indian Journal of Anaesthesia, Medknow Publications \& Media Pvt Ltd, May 2012, www.ncbi.nlm.nih.gov/pmc/articles/PMC3425280/ 